\title{
Developing Additional Hospital Capacity at the Community Level
}

\author{
Ronald Lagoe*, Shelly Littau \\ Hospital Executive Council, Syracuse, USA \\ Email: *Hospexcl@cnymail.com
}

How to cite this paper: Lagoe, R. and Littau, S. (2022) Developing Additional Hospital Capacity at the Community Level. Case Reports in Clinical Medicine, 11, 1-7. https://doi.org/10.4236/crcm.2022.111001

Received: December 1, 2021

Accepted: December 27, 2021

Published: December 30, 2021

Copyright $\odot 2022$ by author(s) and Scientific Research Publishing Inc. This work is licensed under the Creative Commons Attribution International License (CC BY 4.0).

http://creativecommons.org/licenses/by/4.0/

\begin{abstract}
Hospitals in the United States are being challenged to provide the capacity for adult medicine and surgery care. The study suggested that the hospitals of Syracuse, New York have generated additional inpatient capacity through a number of efforts. One program involved moving some low severity of illness inpatient procedures to ambulatory care. A different approach has also avoided inpatient utilization by diverting incoming ambulances to different providers. The third program evaluated in the study, length of stay reduction, was a different type of initiative. It has generated additional inpatient capacity by reducing the amount of inpatient care provided. In effect, it has increased inpatient capacity by addressing the efficiency of care. These programs illustrate the potential for improving hospital capacity at the community level. Each of them was developed by acute care providers using local services.
\end{abstract}

\section{Keywords}

Hospitals, Hospital Emergency Departments, Hospital Lengths of Stay

\section{Introduction}

In the United States, increasing efforts are focusing on the development of hospital inpatient bed capacity. The need for additional inpatient beds has become a major challenge during recent years [1].

One of the most important causes of this need has been the onset of the coronavirus epidemic during 2020 and 2021. The virus has generated the need for additional hospital inpatient capacity to serve adult medicine inpatients. Hospitals in most areas of the nation have been unable to meet this need with existing staff resources [2] [3].

Part of this challenge has been generated by the changing nature of incidence of the virus. At least two waves of virus incidence have developed during 2020 
and 2021. Each of them has been accompanied by substantial increases in the need for inpatient bed capacity.

Reductions in the availability of nursing staff have also challenged the ability of hospitals to operate inpatient capacity. Available evidence suggests that substantial demands on nursing staff time have caused many nurses to reduce their workloads or leave the profession entirely. These problems have apparently not been alleviated by increases in compensation or other benefits [4] [5].

The need for hospitals to provide inpatient acute care has also challenged the operation of other health care services at the community level. These include emergency services through emergency departments and long term care in nursing homes and home health care [6] [7] [8].

This study reviewed examples of efforts to address the need for inpatient care in the hospitals of Syracuse, New York. It identified efforts to improve this situation at the community level, where most inpatient care is delivered.

\section{Population}

This study reviewed recent efforts to develop and support additional inpatient hospital capacity in the metropolitan area of Syracuse, New York. This area includes three large acute care facilities, Crouse Hospital (19,611 inpatient hospital discharges, 2019); St. Joseph's Hospital Health Center (25,394 inpatient discharges, 2019); and Upstate University Hospital (32,877 inpatient discharges, 2019) [9].

These hospitals provide inpatient acute care services to an immediate service area with a population of approximately 600,000 . They also provide referral center services to the eleven county Central New York Health Service Area.

In order to develop additional capacity, the Syracuse hospitals worked with the Hospital Executive Council. This was the planning organization developed by the three providers.

\section{Method}

The programs described in this study comprised a variety of approaches to the development of inpatient hospital capacity. The study focused on results of these programs in the Syracuse hospitals between July and September 2021 compared with the same months in previous years.

The study was based on simple descriptive statistics. Each of the data was based on different indicators.

The initial component of the study involved the development of increases in adult medicine inpatient capacity in two of the Syracuse hospitals through the movement of inpatients to ambulatory care. This program included patients with low severity of illness who were amenable to outpatient settings. For purposes of the study, severity of illness was defined according to the $3 \mathrm{M}^{\mathrm{TM}}$ All $\mathrm{Pa}$ tients Refined Diagnosis Related Group System.

The program focused on inpatients hospitalized for orthopedic surgery in the 
Syracuse hospitals.

This specialty generated large numbers of patients in the hospitals. Within this population, many of these patients were at Minor or Moderate severity of illness.

Evaluation of the program involved comparison of numbers of discharges in the hospitals for July-September 2021 compared with numbers of discharges in the same months in 2017 and 2019. Discharges for all orthopedic surgery and hip and knee joint replacements were identified.

The second component of the study involved the development of additional inpatient capacity in one of the Syracuse hospitals through diversion of incoming ambulance patients to other acute hospitals. This program involved the use of emergency medical services ambulance dispatchers in the Syracuse hospital to direct ambulances to hospitals where additional capacity was available. In the Syracuse area, ambulances were dispatched from the Onondaga County 911 Center and from AMR, the largest ambulance service. Patients' demands for care were honored, regardless of the hospital that was requested.

Evaluation of this program focused comparison of numbers of hours on ambulance diversion for each of the hospitals and numbers of ambulances dispatched. These data were compared for July-September 2017, 2019, and 2021, the most recent time period available. This information provided estimates of the impact of ambulance diversion on hospital emergency department capacity. The numbers of ambulances diverted were not available.

The third component of the study involved the use of hospital length of stay reduction to generate hospital inpatient capacity in the Syracuse hospitals. This approach increased the capacity of the hospitals by reducing the duration of stays and generating the potential for serving additional inpatients. It involved reducing stays for adult medicine and adult surgery, the largest inpatient services in each of the hospitals.

The program was implemented through specific programs addressing patients with potential for extended stays in the hospitals. They included the Subacute and Complex Care Programs, which supported efforts by nursing homes to admit patients with extended intravenous services and wound care in the hospitals through program development funds. It was also supported through the weekly distribution of community wide data concerning Difficult to Place Patients in the community. Evaluation of the length of stay reduction program involved comparison of inpatient adult medicine and adult surgery lengths of stay in the hospitals for 2021, 2017, and 2019. Using this information, it was possible to quantify the additional amounts of inpatient capacity that were made available.

\section{Results}

The initial component of the study involved increasing hospital capacity by moving utilization to outpatient services. Relevant data are summarized in Table 1 . 
Table 1. Inpatient hospital discharges by severity of illness, Syracuse Hospitals, July-September 2107, 2019, 2021.

\begin{tabular}{cccccc}
\hline & \multicolumn{5}{c}{ Orthopedic Surgery (APR DRGs 303 - 322) } \\
\hline & Minor & Moderate & Major & Extreme & Total \\
\hline July-September 2017 & 381 & 488 & 150 & 21 & 1040 \\
July-September 2019 & 364 & 466 & 171 & 39 & 1040 \\
July-September 2021 & 260 & 386 & 164 & 56 & 866 \\
Difference 2017-2021 & -121 & -102 & 14 & 35 & -174 \\
\hline & Hip \& Knee Joint Replacement (APR DRGs 301 - 302) \\
\hline & Minor & Moderate & Major & Extreme & Total \\
\hline July-September 2017 & 516 & 408 & 37 & 10 & 971 \\
July-September 2019 & 392 & 426 & 33 & 10 & 861 \\
July-September 2021 & 153 & 210 & 31 & 17 & 411 \\
Difference 2017-2021 & -363 & -198 & -6 & 7 & -560 \\
\hline
\end{tabular}

Data include patients aged 18 years and over. Source: Hospital Executive Council.

This information demonstrated that between July-September 2017 and 2021, the Syracuse hospitals reduced the number of orthopedic inpatient discharges by 174. During the same period, the hospitals reduced the number of hip and knee joint replacement discharges by 560 . The joint replacement patients that were eliminated would have generated 1465 patient days or an average daily census of 15.92 patients.

The study data also demonstrated that most of the reductions in inpatient utilization involved hospital inpatients with low severity of illness. For orthopedics, the reduction in discharges involved 121 Minor severity of illness and 102 Moderate severity of illness inpatients. For the hip and knee joint replacements, the change involved a reduction in discharges of 363 Minor severity of illness and 198 Moderate severity of illness inpatients.

Between July and September 2017 and 2021, much smaller changes occurred in orthopedic surgery and joint replacements at Major and Extreme severity of illness. Most of these changes resulted in slight increases in inpatient discharges.

Information related to the study data suggested that most of these changes resulted from the movement of hospital inpatients to ambulatory care by orthopedic surgeons. Regardless of the source of the changes, they made additional inpatient capacity available in the hospitals.

The second component of the study involved the development of additional inpatient capacity through ambulance diversion from emergency departments in the Syracuse hospitals. Related data are summarized in Table 2.

This information demonstrated that the use of ambulance diversion in the Syracuse hospitals increased substantially between July and September 2017 and 2021. The number of hours on ambulance diversion rose by 2474 between these time periods. 
Table 2. Hospital emergency department utilization data, Syracuse Hospitals, July-September 2017, 2019, 2021.

\begin{tabular}{lcc}
\hline & Hours on Ambulance Diversion & Ambulance Transports \\
\hline July-September 2017 & 124 & 15,666 \\
July-September 2019 & 285 & 16,899 \\
July-September 2021 & 2598 & 17,331 \\
Difference 2017-2021 & 2474 & 1665 \\
\hline
\end{tabular}

Source: Hospital Executive Council.

Of the total hours on diversion, $1670,67.5$ percent involved one of the hospitals. This hospital employed the mechanism to shift adult medicine inpatients to other facilities. By increasing the use of ambulance diversion, the hospital increased capacity available for other services, such as elective surgery and treatment of patients with the Covid virus.

The impact of this change on inpatient utilization was limited by the fact that this hospital continued to admit some patients for services such as stroke and burn care. It was also limited somewhat by the fact that patient demands for each transport to each of the hospitals continued to support existing utilization patterns.

The third component of the study involved the use of length of stay reduction to increase inpatient capacity in the Syracuse hospitals. Relevant data are summarized in Table 3.

The comparisons of inpatient lengths of stay among the three hospitals demonstrated that these initiatives could generate substantial differences in utilization.

Table 3. Inpatient adult medicine and adult surgery mean length of stay (Days) by severity of illness, Syracuse Hospitals, July-September 2021.

\begin{tabular}{ccccccccccc}
\hline & \multicolumn{4}{c}{ Adult Medicine } & \multicolumn{5}{c}{ Adult Surgery } \\
\hline & Minor & Moderate & Major & Extreme & Total & Minor & Moderate & Major & Extreme & Total \\
\hline Mean Lengths of Stay & & & & & & & & & \\
Hospital A & 2.63 & 3.91 & 6.17 & 11.19 & 5.89 & 2.35 & 4.23 & 8.79 & 16.37 & 5.46 \\
Hospital B & 2.23 & 3.06 & 5.01 & 9.17 & 4.70 & 2.16 & 3.60 & 6.90 & 14.34 & 5.43 \\
Hospital C & 2.89 & 3.71 & 5.35 & 10.16 & 5.45 & 2.83 & 4.19 & 8.62 & 18.46 & 7.26 \\
Total & 2.62 & 3.55 & 5.43 & 10.12 & 5.32 & 2.41 & 4.00 & 7.93 & 16.58 & 6.17 \\
\hline Number of Discharges & & & & & & & & & & \\
Hospital A & 252 & 762 & 809 & 397 & 2220 & 381 & 341 & 150 & 101 & 973 \\
Hospital B & 345 & 980 & 1138 & 472 & 2935 & 521 & 574 & 409 & 241 & 1745 \\
Hospital C & 476 & 1341 & 1643 & 790 & 4250 & 354 & 766 & 429 & 297 & 1846 \\
Total & 1073 & 3083 & 3590 & 1659 & 9405 & 1256 & 1681 & 988 & 639 & 4564 \\
\hline
\end{tabular}

Adult medicine data exclude Diagnosis Related Groups concerning surgery, obstetrics, psychiatry, alcohol/substance abuse treatment, rehabilitation, and all patients aged 0 - 17 years. Adult surgery data exclude Diagnosis Related Groups concerning surgery, obstetrics, psychiatry, alcohol/substance abuse treatment, and all patients aged 0 - 17 years. Source: Hospital Executive Council. 
Separate analyses were developed for adult medicine and adult surgery. These differences generated opportunities for additional inpatient capacity.

This information demonstrated that inpatient adult medicine lengths of stay for one of the providers, Hospital B, were shorter than those of the other hospitals for all four levels of severity of illness. The lengths of stay for Hospital B were 1.19 days shorter than those of Hospital A. This difference amounted to 3493 patient days or an average daily census of 38.0 patients. The lengths of stay for Hospital B were 0.7 days shorter than those of Hospital C. This difference amounted to 2201 patient days or an average daily census of 23.9 patients.

This information also demonstrated that the adult surgery lengths of stay for Hospital B were shorter than those of the other hospitals for all four levels of severity of illness. The stays for Hospital B were 0.3 days shorter than those of Hospital A. This difference amounted to 52.4 patient days or an average daily census of 0.6 patients. The adult surgery stays for Hospital B were 1.83 days shorter than those of Hospital C. This amounted to 3193 patient days or an average daily census of 34.7 patients.

All of these differences produced increased inpatient utilization. They could be translated into capacity for additional inpatient services.

\section{Discussion}

Hospitals in the United States are being confronted with epidemics, staffing shortages, and the increased referrals from other services. They are being challenged to provide the capacity for adult medicine and surgery care. This study provided examples of efforts to address these needs in one community.

The study suggested that the hospitals of Syracuse, New York have generated additional inpatient capacity through a number of efforts. The most basic programs involved addressing the need by moving some low severity of illness inpatient procedures to ambulatory care. In the examples provided in this study, this approach was supported by physicians who developed their own programs to treat orthopedic patients.

A different approach has also avoided inpatient utilization by diverting incoming ambulances to different providers. These initiatives have created capacity for additional inpatients by moving some of them to other settings.

The third program evaluated in the study, length of stay reduction, was a different type of initiative. It has generated additional inpatient capacity by reducing the amount of inpatient care provided. In effect, it has increased inpatient capacity by addressing the efficiency of care. It has reduced numbers of inpatient acute care days without reducing numbers of hospital inpatients.

In the Syracuse hospitals, length of stay reduction was supported by cooperative efforts with area nursing homes. These programs shifted patient days from hospitals to long term care facilities.

These programs illustrate the potential for improving hospital capacity at the community level. Each of them was developed by acute care providers using lo- 
cal services.

\section{Conflicts of Interest}

The authors declare there are no conflicts of interest regarding the publication of this paper.

\section{References}

[1] Dentzler, S. (2011) Urgent Measures for an Old Problem. Health Affairs, 30, 1626. https://doi.org/10.1377/hlthaff.2011.0961

[2] Goozner, M. (2020) Big Data's Role in Addressing COVID-19. Modern Healthcare, $50,22$.

[3] Johnson, S. (2021) Will COVID-19 Be the Catalyst for Creating a More Sustainable Health Care System? Modern Healthcare, 51, 12-14.

[4] Weil, A.R. (2015) Hospital Costs and Quality. Health Affairs, 34, 1263. https://doi.org/10.1377/hlthaff.2015.0786

[5] Livingston, S. (2020) Providers Sideline Employees, Cut Pay as Pandemic Saps Revenue. Modern Healthcare, 50, 6-7.

[6] Cushing, W.T. (2004) Extra Hospital Days Can Cost You Plenty. Medical Economics, 81, 83 .

[7] Gawande, A.A. (2011) The Hot Spotters: Can We Lower Costs by Giving the Neediest Patients Better Care? New Yorker.

[8] Oriol, W. (1985) The Complex Cube of Long Term Care. American Health Planning Association, Washington D.C.

[9] Lagoe, R., Pasinski, T., Kronenberg, P., Quinn, T. and Schaengold, P. (2006) Linking Health Services at the Community Level. Canada Health Care Quarterly, 9, 60-65. https://doi.org/10.12927/hcq..18229 